\title{
Recomendaciones para el abordaje y tratamiento de la intolerancia a la glucosa
}

\section{Glucose intolerance management and treatment recommendations}

\author{
Paloma Almeda-Valdes ${ }^{1,2 *}$, Sergio Hernández-Jiménez ${ }^{3}$, Francisco J. Gómez-PÉrez ${ }^{1}$, Ana C. García UlloA ${ }^{3}$, \\ Rafael Campuzano-Rodríguez ${ }^{4}$, Miguel A. Madero-FÉRnÁNdez ${ }^{5}$, Natalia E. de la Garza-Hernández \\ Josefina B. Cota-Aguilar 7 , SARA Arellano-Montaño ${ }^{8}$ y Fernando J. Lavalle-GonzÁlez ${ }^{9}$
}

'Departamento de Endocrinología y Metabolismo. Instituto Nacional de Ciencias Médicas y Nutrición Salvador Zubirán, Ciudad de México; ${ }^{2}$ Unidad de Investigación de Enfermedades Metabólicas, Instituto Nacional de Ciencias Médicas y Nutrición Salvador Zubirán, Ciudad de México; ${ }^{3}$ Centro de Atención Integral del Paciente con Diabetes, Instituto Nacional de Ciencias Médicas y Nutrición Salvador Zubirán, Ciudad de México; ${ }^{4}$ Departamento de Endocrinología, Universidad Michoacana, Morelia, Mich.; ${ }^{5}$ Facultad de Medicina, Universidad Autónoma de Coahuila, Torreón, Coah.; ${ }^{6}$ Departamento de Endocrinología, Centro Médico Integral (CEMEDIN), San Pedro Garza García, Monterrey N.L.; ${ }^{7}$ Practica Privada, Tijuana, B.C.; ${ }^{8}$ Servicio de Endocrinología, Hospital General de México, Ciudad de México; ${ }^{9}$ Facultad de Medicina, Hospital Universitario “Dr. José E. González”, Universidad Autónoma de Nuevo León (UANL), Monterrey, N.L. México

\section{RESUMEN}

La prediabetes es una condición caracterizada por alteraciones en el metabolismo de la glucosa que incluye a la glucosa alterada en ayuno, la intolerancia a la glucosa y la elevación de hemoglobina glucosilada. Estas alteraciones, además de conferir un riesgo incrementado para el desarrollo de diabetes, aumentan el riesgo de desarrollar complicaciones microvasculares y macrovasculares. La detección y tratamiento de estas alteraciones en los casos con riesgo son estrategias necesarias que pueden disminuir la carga asociada a la diabetes y enfermedad cardiovascular.

Palabras clave: Intolerancia a la glucosa. Glucosa alterada en ayuno. Hemoglobina glucosilada.

\begin{abstract}
Prediabetes is a condition due to alterations in glucose metabolism that includes abnormal fasting glucose, glucose intolerance and glycated hemoglobin elevation. These alterations confer an increased risk for diabetes and micro and macrovascular complications development. Detection and treatment of these alterations in patients at risk are strategies that can lower the burden associated with diabetes and cardiovascular disease.
\end{abstract}

Key words: Glucose intolerance. Abnormal fasting glucose. Glycated hemoglobin.
Correspondencia:

*Paloma Almeda-Valdés

E-mail: paloma.almedav@incmnsz.mx
Fecha de recepción: 26-11-2019

Fecha de aceptación: 11-01-2020

DOI: 10.24875/RME.20001965
Disponible en internet: 03-11-2020

Rev Mex Endocrinol Metab Nutr. 2020;7:202-11

2462-4144 / @ 2020 Sociedad Mexicana de Nutricion y Endocrinologia, AC. Publicado por Permanyer. Éste es un artículo open access bajo la licencia CC BY-NC-ND (http://creativecommons.org/licenses/by-nc-nd/4.0/). 


\section{INTRODUCCIÓN}

En la historia natural de la diabetes tipo 2 (DT2) no es posible la identificación precisa del momento en el cual inician las alteraciones metabólicas, ya que los síntomas son muy insidiosos y heterogéneos. Por ello existen los puntos de corte en diferentes parámetros validados en estudios epidemiológicos y en estudios prospectivos que han relacionado estos puntos con complicaciones crónicas. Los puntos de corte para considerar a un individuo con prediabetes han sido algo arbitrarios y basados en el grado de riesgo de progresión a diabetes. La DT2 es una de las crisis de salud pública más devastadora, por lo que es necesario enfocar los esfuerzos a su prevención y la de sus complicaciones. Esta prioridad hace dirigir la atención a identificar en forma temprana a los individuos en riesgo y a establecer medidas terapéuticas oportunas. El objetivo de esta propuesta es difundir las acciones prácticas aplicables en cualquier entorno y por cualquier profesional de la salud para identificar alteraciones tempranas en el metabolismo de la glucosa y promover el abordaje de mayor impacto para el tratamiento de la DT2: la prevención.

\section{DEFINICIÓN Y DIAGNÓSTICO DE} INTOLERANCIA A LA GLUCOSA

El término prediabetes se refiere a un estado metabólico en el cual la concentración de glucosa no es suficientemente alta para establecer el diagnóstico de diabetes pero tampoco es normal'. Incluye las siguientes condiciones:

- Glucemia de ayuno entre 100 y 125 mg/dl después de un ayuno de $12 \mathrm{~h}$ (glucosa alterada en ayuno).

- Glucemia entre 140 y 199 mg/dl 2 h después de una toma de $75 \mathrm{~g}$ de glucosa por vía oral (intolerancia a la glucosa $[I G])$.

- Hemoglobina glucosilada (HbA1c) entre 5.7 y el $6.4 \%$.

Estas categorías no son equivalentes: tener glucemia alterada de ayuno no es equivalente a IG. EI estudio Euro Heart ${ }^{1}$ reportó que la prueba de curva de tolerancia oral a la glucosa (CTGO) detectó a un número significativamente mayor de individuos con alteraciones al que podría ser detectado solo con la medición de la glucemia en ayunas.

¿CÓMO HACER EL TAMIZAJE TEMPRANO?

Recabar los siguientes datos prioritarios en personas mayores de 20 años:

- Presencia de algún familiar de primer grado (padre, hermano, hijo) con DT2.

- Diagnóstico previo de diabetes gestacional.

- Mujer que haya tenido un producto macrosómico (> $4 \mathrm{~kg}$ al nacer).

- Enfermedad cardiovascular (infarto de miocardio, enfermedad vascular cerebral, enfermedad arterial periférica).

- Hipertensión arterial sistémica.

- Índice de masa corporal (IMC) $>25 \mathrm{~kg} / \mathrm{m}^{2}$.

- Triglicéridos > $150 \mathrm{mg} / \mathrm{dl}$.

- Tabaquismo.

- Hígado graso.

- Síndrome de ovario poliquístico.

- Acantosis nigricans y/o acrocordones.

En presencia de uno o más de estos elementos, está indicado medir la glucemia de ayuno. Si el resultado es $<100 \mathrm{mg} / \mathrm{dl}$, se recomienda la medición de glucosa en ayuno al menos cada tres años. Si se reporta un valor entre 100 y $125 \mathrm{mg} / \mathrm{dl}$, se recomienda realizar una CTOG de $2 \mathrm{~h}$ (Fig. 1).

\section{PRUEBA DE TOLERANCIA A LA GLUCOSA ORAL}

Este estudio dinámico refleja la habilidad de las células beta del páncreas para secretar insulina y la 


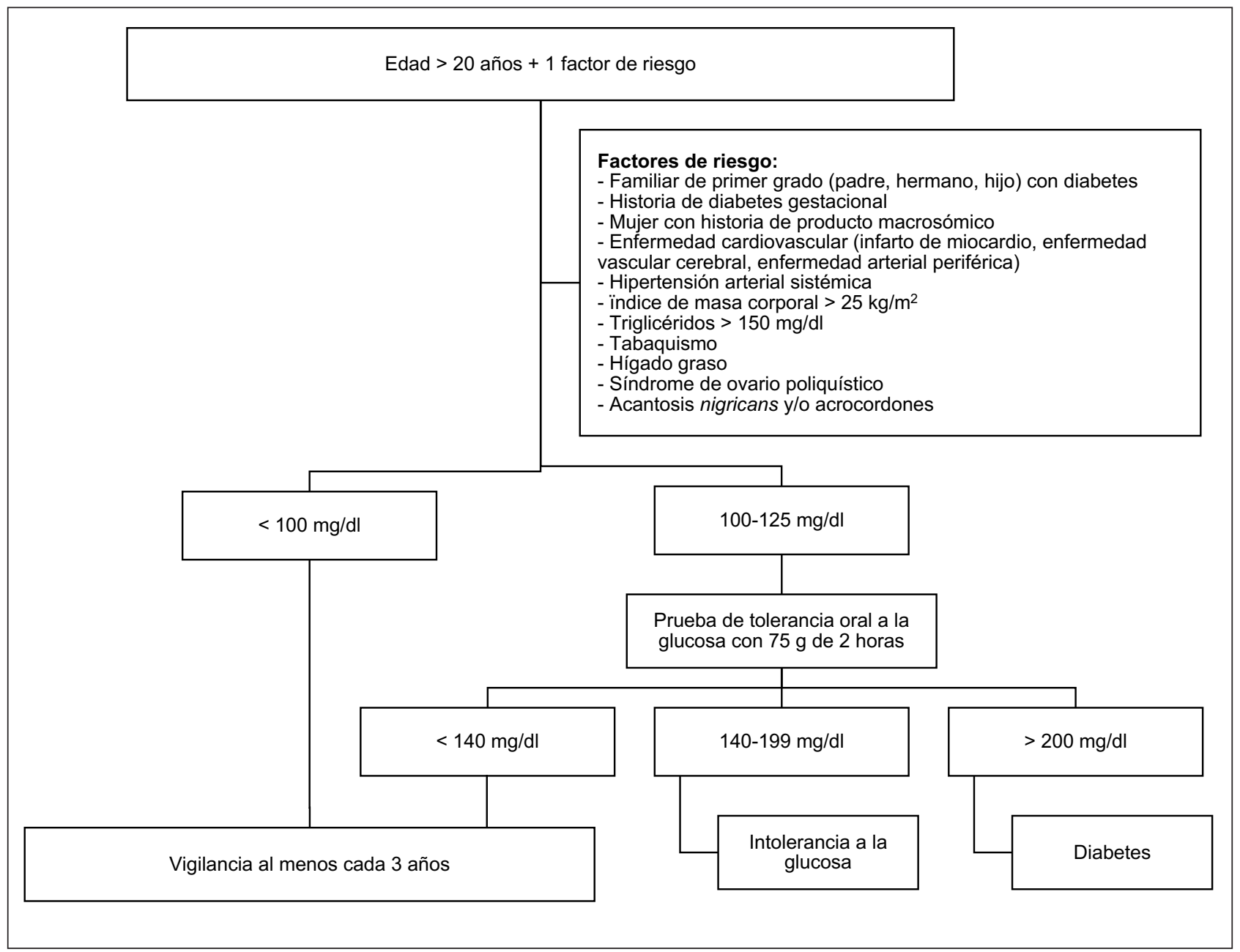

Figura 1. Algoritmo de decisión para abordar oportunamente el diagnóstico de alteraciones de la glucemia.

sensibilidad de los tejidos a la insulina. La CTGO con determinación de glucosa en ayuno y a las dos horas es un estudio que se debe realizar en toda persona que presente una glucemia de ayuno entre 100 y $125 \mathrm{mg} / \mathrm{dl}$. Si el resultado se encuentra entre 140 y $199 \mathrm{mg} / \mathrm{dl}$, se establece el diagnóstico de IG y si la concentración de glucosa a las dos horas es $>200$ $\mathrm{mg} / \mathrm{dl}$, se establece el diagnóstico de diabetes.

Se puede realizar la CTGO en personas con glucemia de ayuno $>95 \mathrm{mg} / \mathrm{dl}$ con alguno de los siguientes factores de riesgo ${ }^{2}$ :

- Familiar de primer grado con diagnóstico de DT2.

- Historia de diabetes gestacional o haber tenido hijo o hija $\geq 4 \mathrm{~kg}$ al nacer.
- IMC $\geq 25 \mathrm{~kg} / \mathrm{m}^{2}$.

- Presencia de acantosis nigricans.

- Hipertensión arterial sistémica.

- Triglicéridos > $150 \mathrm{mg} / \mathrm{dl}$.

La realización de variantes de la prueba, por ejemplo con determinaciones de glucosa basal, a los 30, 60,90 y 120 minutos y la prueba de cinco horas de duración pueden ser de utilidad en casos particulares, por ejemplo en personas en quienes presentan datos clínicos de hipoglucemia posprandial (a las 3 a 5 h) y en estudios de investigación.

Se ha postulado la medición de la concentración de insulina en ayuno como indicador de resistencia a la insulina. En un estudio que incluyó 258 voluntarios 
Tabla 1. Procedimiento y consideraciones para realizar una CTOG

\begin{tabular}{ll}
$\begin{array}{l}\text { Preparación previa } \\
\text { al estudio }\end{array}$ & Ayuno de 8 a 12 h \\
\hline $\begin{array}{l}\text { Cuidados durante la } \\
\text { prueba }\end{array}$ & $\begin{array}{l}\text { Se recomienda llevar una alimentación habitual en los días previos } \\
\text { No debe hacerse en personas con glucosa en ayuno } \geq 126 \mathrm{mg} / \mathrm{dl}\end{array}$ \\
& $\begin{array}{l}\text { Permanecer en reposo durante la prueba } \\
\text { Al término de la prueba es importante desayunar }\end{array}$ \\
\hline Riesgos & Esta prueba NO causa diabetes \\
& Ocasionalmente puede asociarse con náuseas, vómitos, dolor de cabeza o mareo \\
\hline Procedimiento & 1. Se toma una muestra de glucosa en sangre \\
& $\begin{array}{l}\text { 2. Se le da al paciente a beber una solución de glucosa (75 g en 250 ml) } \\
\text { 3. Durante la prueba el paciente debe estar en reposo y no comer }\end{array}$ \\
\hline 4. Dos horas después de ingerir la solución, se toma otra muestra de glucosa en sangre \\
invalidan la prueba & 2. Vómitos \\
& 3. Los pacientes desnutridos o en cama pueden dar resultados falsos positivos \\
& 4. La presencia de anorexia o cualquier otra causa que impida una ingesta adecuada invalida la prueba \\
\hline
\end{tabular}

CTOG: curva de tolerancia oral a la glucosa.

con sobrepeso, sin diabetes y sin hipertensión, en quienes se realizó una prueba de supresión con insulina para identificar a individuos con resistencia a la insulina, el mejor punto de corte de insulina en ayuno para identificar a individuos con resistencia a la insulina fue de $15.7 \mu \mathrm{U} / \mathrm{ml}$, con una sensibilidad del $57 \%$ y especificidad del $85 \%{ }^{3}$. Sin embargo, la medición de la insulina tanto en ayuno como durante la CTOG se ve limitada por la gran cantidad de ensayos disponibles con diferente rendimiento y la falta de estandarización de estos.

\section{PROCEDIMIENTO Y TÉCNICA ADECUADOS PARA REALIZAR UNA CTOG}

El procedimiento para realizar una CTGO se describe en la tabla 1.

\section{¿QUÉ IMPLICACIONES CLÍNICAS TIENE LA INTOLERANCIA A LA GLUCOSA?}

Además de estar asociada a una mayor incidencia de diabetes, la IG también se asocia al desarrollo de complicaciones microvasculares y macrovasculares.
Un metaanálisis de 26 estudios prospectivos reportado en 2014 que incluyó más de 280,000 individuos mostró que los individuos con IG tienen un riesgo relativo (RR) de 1.33 (intervalo de confianza [IC] 95\%: 1.24-1.42) de mortalidad por todas las causas y de 1.23 (IC 95\%: 1.11-1.36) de mortalidad cardiovascular ${ }^{4}$. En un metaanálisis posterior ${ }^{5}$, el mismo grupo conjuntó información de 53 estudios para evaluar el riesgo en glucosa alterada en ayuno, IG y HbA1c, y concluyó que la prediabetes definida por los tres conceptos se asocia a incremento del riesgo de enfermedad cardiovascular y que el riesgo podría incrementarse desde concentraciones de glucosa en ayuno de $100 \mathrm{mg} / \mathrm{dl}$ y $\mathrm{HbA} 1 \mathrm{c}$ de $5.7 \%$. En un reporte de individuos mayores de 50 años con prediabetes extraídos de la cohorte Whitehall II ${ }^{6}$ se encontró que la tasa de incidencia de enfermedad cardiovascular fatal y no fatal o mortalidad por cualquier causa fue un $37 \%$ mayor (RR: 1.37 ; IC 95\%: 1.20-1.57) en pacientes con prediabetes (glucosa alterada en ayuno, IG o HbA1c elevada) en comparación con individuos sin prediabetes, pero se redujo al 12\% (RR: 1.12; IC 95\%: 0.97-1.28), perdiendo significancia estadística después del ajuste para distintos confusores (edad, sexo, etnicidad, enfermedad cardiovascular previa, tabaquismo, colesterol total, colesterol HDL [high density lipoproteins], presión arterial sistólica y tratamiento antihipertensivo). 
El estudio $\mathrm{ARIC}^{7}$ integró entre 1987 y 1989 una cohorte de 15,792 individuos en EE.UU. con seguimientos trianuales. Las mediciones de $\mathrm{HbA} 1 \mathrm{c}$, glucosa en ayuno y poscarga fueron confrontadas con desenlaces que incluían diabetes, enfermedad renal crónica, enfermedad ateroesclerótica (cardiovascular, cerebrovascular y periférica) y mortalidad por todas las causas. El estudio demostró que un valor de $\mathrm{HbA} 1 \mathrm{c}$ por arriba del $5.5 \%$ incrementa significativamente el riesgo de enfermedad coronaria.

TRATAMIENTO DE LA INTOLERANCIA A LA GLUCOSA

\section{Cambios en el estilo de vida: plan de alimentación y actividad física}

La progresión a DT2 en personas con prediabetes no es inevitable. La pérdida de peso modesta y sostenida, mediante terapia nutricional individualizada, y el aumento en la actividad física y/o tratamiento con metformina pueden prevenir o retardar la aparición de la diabetes.

El Programa de Prevención de Diabetes (DPP) ${ }^{8}$ y el Programa de Prevención de Diabetes de Finlandia ${ }^{9}$ lograron una pérdida de peso promedio del 7 y el $5 \%$, respectivamente, en participantes con IG asignados a una intervención con cambios en el estilo de vida. En ambos estudios se observó reducción de la incidencia de diabetes de hasta un 58\% en comparación con placebo en tres años y en el DPP en personas mayores hasta un $71 \%$ en tres años. En el seguimiento a 10 años del estudio DPP se encontró que la intervención con cambios en el estilo de vida redujo la aparición de diabetes en un $34 \%$ (retardando su aparición alrededor de cuatro años) y redujo su aparición en un $18 \%$ en el grupo de metformina, comparados con placebo ${ }^{10}$.

Si bien las modificaciones en el estilo de vida son el método más efectivo para reducir el riesgo de diabetes, la mayoría de las personas no las llevan a cabo. Esto indica una fuerte necesidad de programas de educación.
Haw, et al. ${ }^{11}$ estimaron los efectos a largo plazo de diferentes estrategias de prevención de diabetes en adultos en riesgo. Se incluyeron datos de 43 estudios con 49,029 participantes que evaluaron modificaciones en el estilo de vida (19 estudios), intervención con medicamentos por más de seis meses (19 estudios) y la combinación de ambas estrategias (5 estudios) para la prevención de la diabetes en adultos. Los investigadores encontraron que las modificaciones en el estilo de vida se asociaron con una reducción del RR de diabetes del 39\% y los medicamentos con una reducción de RR del 36\%; sin embargo, al final de la intervención activa los estudios con medicamentos no mostraron una reducción sostenida.

Wilmot, et al. ${ }^{12}$, en un metaanálisis sobre vida sedentaria y salud, combinaron los resultados de 18 estudios incluyendo un total de 794,577 participantes (16 estudios eran prospectivos). El mayor tiempo sedentario comparado con el menor se asoció con aumento en dos veces del riesgo de diabetes (RR: 2.12).

\section{Terapia nutricional}

El paciente debe recibir valoración y terapia nutricional individualizada con reducción de la ingesta de calorías. Los siguientes conceptos son recomendaciones generales:

- Grasas: las grasas saturadas deben evitarse o limitarse a menos del $10 \%$ del total de las calorías. Las grasas trans se deben evitar y se recomienda elegir grasas monoinsaturadas y poliinsaturadas (incluyendo ácidos grasos omega-3) como pescado, salmón, nueces, semillas, aguacate y aceites (oliva, canola, girasol).

- Carbohidratos: seleccionar los de alto contenido en fibra y limitar alimentos con azúcares agregados y azúcares refinados.

- Proteínas: deben representar el 15-20\% de las calorías. Escoger fuentes animales bajas en grasas y de origen vegetal (frijoles, garbanzo).

- Fibra: consumir $15 \mathrm{~g}$ por cada 1,000 kcal.

- Sodio: limitar la ingesta a 1,500-2,300 mg/día.

- Alcohol: limitar a 2 porciones/día para hombres y 1/día para mujeres. 
Se sugiere incluir alimentos que se han asociado con disminución del riesgo de diabetes, por ejemplo: nueces, frutos del bosque, yogurt, café y té, y por otra parte evitar los alimentos que se han asociado con el riesgo de diabetes, como carne roja y bebidas azucaradas $^{13}$.

\section{Actividad física}

Como medida inicial, es importante reducir el sedentarismo. Se recomienda el uso de podómetros para determinar el número de pasos que se realicen al día, idealmente logrando un promedio de 10,000 pasos al día y evitando que se realicen menos de 5,000 pasos al día.

Se sugiere recomendar actividad física (aeróbica y de resistencia) de moderada a intensa por un mínimo de 150 minutos por semana.

Frecuentemente se utilizan los equivalentes metabólicos (MET) para expresar la intensidad de la actividad física. Los MET son la razón entre el metabolismo de una persona durante la realización de un trabajo y su metabolismo basal. Un MET se define como el gasto energético de estar sentado tranquilamente y es equivalente a un consumo de $1 \mathrm{kcal} / \mathrm{kg} / \mathrm{h}$. Se calcula que, en comparación con esta situación, el consumo calórico es unas 3 a 6 veces mayor (3-6 MET) cuando se realiza una actividad de intensidad moderada, y más de 6 veces mayor ( $>6 \mathrm{MET}$ ) cuando se realiza una actividad vigorosa.

La actividad física moderada requiere un esfuerzo, que acelera de forma perceptible el ritmo cardiaco. Algunos ejemplos de ejercicio moderado son: caminar a paso rápido, bailar, realizar jardinería, tareas domésticas, participación activa en juegos y deportes con niños y paseos con animales domésticos, etc.

La actividad física intensa (aproximadamente $>6 \mathrm{MET}$ ) requiere una gran cantidad de esfuerzo y provoca una respiración rápida y un aumento sustancial de la frecuencia cardiaca. Se consideran ejercicios vigorosos: ascender a paso rápido o trepar por una ladera, desplazamientos rápidos en bicicleta, aeróbicos, natación rápida, deportes y juegos competitivos (p. ej., juegos tradicionales, fútbol, voleibol, hockey, baloncesto), etc ${ }^{14}$.

Es importante evaluar al paciente en caso de contraindicaciones o limitaciones y elaborar un plan gradual ${ }^{13}$ :

1) Aeróbica: de suficiente intensidad y duración en episodios mínimos de 10 minutos.

2) Resistencia: actividad de beneficio para aumentar fuerza muscular. Por ejemplo: levantar pesas (se recomienda dos días a la semana, hasta 12 repeticiones, 2 a 3 veces para cada grupo muscular), tenis en competencia, bandas de resistencia, lagartijas.

Los programas para lograr pérdida de peso son efectivos para lograr los cambios en el estilo de vida asociados con la prevención de diabetes, pueden tener un impacto significativo en la salud pública de forma inmediata y sobre todo a largo plazo.

\section{Fármacos, dosis y duración}

El tratamiento farmacológico de la IG tiene como objetivo mejorar la sensibilidad a la insulina y promover la pérdida de peso, intentando evitar la progresión a DT2. El fármaco utilizado por excelencia en el tratamiento de la IG es la metformina, la cual ha demostrado reducir la progresión a diabetes. La dosis utilizada en el estudio DPP ${ }^{15}$ fue $850 \mathrm{mg}$ dos veces por día. Los efectos colaterales asociados a este fármaco son molestias gastrointestinales tales como náuseas, vómitos, meteorismo y/o diarrea, los cuales se presentan en el $10 \%$ de los pacientes y pueden reducirse a la mitad de los casos utilizando presentaciones de liberación prolongada.

Otros fármacos que pudieran utilizarse para la prevención de diabetes en personas con IG incluyen:

1) Orlistat: inhibidor de la lipasa gástrica y pancreática que promueve una pérdida de peso del 2.9$3.4 \%$, suele provocar efectos secundarios gastrointestinales como esteatorrea, flatulencia, incontinencia fecal y disminución de la absorción de vitaminas liposolubles. La dosis es entre 60 y $120 \mathrm{mg}$ tres veces por día ${ }^{16}$ En el estudio 
XENDOS (XENical in the prevention of Diabetes in Obese Subjects) se asoció con una reducción del RR del $37 \%$ de progresión a diabetes $(p=0.0032)$ en un seguimiento de cuatro años ${ }^{17}$.

2) Fentermina: agente liberador de norepinefrina aprobado para su uso en periodos cortos, de tres meses. Promueve una pérdida de peso promedio de $3.6 \mathrm{~kg}$, suele asociarse con efectos colaterales como insomnio, cefalea, boca seca, taquicardia y en algunos casos hipertensión. Dosis: 7.5-15 mg por día. La fentermina se ha combinado en EE.UU. con topiramato en dosis de $3.75 \mathrm{mg} / 23 \mathrm{mg}$ a $15 \mathrm{mg} / 19 \mathrm{mg}$, promoviendo la modulación del receptor del ácido $\gamma$-aminobutírico y duplicando la pérdida de peso. Existe un estudio ${ }^{18}$ en el que se utilizó fentermina junto con canagliflozina $300 \mathrm{mg} / \mathrm{día}$ (un inhibidor del cotransportador sodio-glucosa tipo 2), con pérdida de peso significativa.

3) Liraglutida: agonista del receptor de péptido 1 similar al glucagón que promueve pérdida de peso con un promedio de $5.8 \mathrm{~kg}$ a un año de uso. Tiene como efectos colaterales náuseas y vómitos. Dosis escalonada ascendente: de 0.6 a $3 \mathrm{mg}^{16}$. En un estudio, la liraglutida a dosis de $3 \mathrm{mg} /$ día se asoció con prolongación del tiempo para desarrollar diabetes a tres años, sin embargo, un $47 \%$ de los participantes en el grupo de liraglutida y un $55 \%$ en el grupo de placebo abandonaron el estudio ${ }^{19}$.

4) Inhibidores de la alfa-glucosidasa: en el estudio STOP-NIDDM (Study TO Prevent Non Insulin Dependent Diabetes Mellitus) el tratamiento con acarbosa a dosis de $100 \mathrm{mg}$ tres veces al día se asoció con disminución del riesgo de diabetes (RR: 0.75; IC 95\%: 0.63-0.90; $p=0.0015$ ), sin embargo, el 31\% de los participantes asignados a acarbosa abandonaron el estudio por eventos adversos como flatulencia y diarrea ${ }^{20}$.

5) Tiazolidinedionas: en un estudio con pioglitazona en individuos con IG con seguimiento de 2.4 años, el hazard ratio (HR) para la conversión de diabetes fue 0.28 (IC 95\%: 0.16-9.49; $p<0.001$ ) vs. placebo. Sin embargo, el aumento de peso y el edema fueron más frecuentes en el grupo asignado a pioglitazona ${ }^{21}$.
Salud mental: etapa de duelo y motivación al cambio

\section{Duelo}

El duelo es un proceso de adaptación emocional ante cualquier pérdida (en este caso, la pérdida de la salud). Aunque convencionalmente se ha enfocado a la respuesta emocional, el duelo también incluye el comportamiento humano. Se han descrito cinco etapas que caracterizan al proceso del duelo:

1) Negación: la incredulidad ante lo ocurrido es la reacción más común. En este punto hay resistencia o ausencia de cambio.

2) Ira: buscar un culpable de lo ocurrido. Hay resistencia a llevar a cabo las indicaciones sugeridas por los profesionales de la salud y en ocasiones hay hostilidad.

3) Negociación: aceptación de la enfermedad, poniendo condiciones para seguir el tratamiento y con apego parcial.

4) Tristeza: el paciente nota lo que ha perdido (su salud), por lo que puede presentarse con apatía o poca motivación.

5) Aceptación: el paciente entiende su situación y genera estrategias para apegarse.

\section{Motivación al cambio}

Se describen cinco etapas del cambio que permiten entender cuándo ocurre:

1) Precontemplación: no hay intención de cambiar en los próximos seis meses. En esta etapa la persona no le da importancia a su problema. Es el caso del paciente acude a buscar tratamiento por presión y después lo abandona.

2) Contemplación: la persona es consciente de que tiene un problema, pero no se compromete a cambiar. El cambio se prevé en los siguientes seis meses. La persona le ve ventajas a mantener la conducta problema.

3) Preparación: se planean cambios en el mes siguiente. Generalmente la persona ha tenido 
pocos cambios en el último año, pero aún no alcanza el cambio.

4) Acción: los individuos modifican su comportamiento. El cambio es de entre un día y seis meses, buscando alcanzar el criterio establecido.

5) Mantenimiento: se trabaja para prevenir recaídas y consolidar ganancias. Esta etapa debe durar más de seis meses manteniéndose libre de la conducta problema.

\section{SEGUIMIENTO DE LA EFECTIVIDAD DEL TRATAMIENTO}

El monitoreo de la prediabetes para detectar empeoramiento en la alteración de la glucosa debe incluir la realización anual de CTOG de dos horas con $75 \mathrm{~g}$ de glucosa, glucosa en ayuno y HbA1c. El perfil de lípidos debe determinarse al menos dos veces por año. Los pacientes con riesgo mayor deben de ser monitoreados más estrechamente y en presencia de hiperglucemia marcada o alteración de los parámetros de riesgo cardiovascular es necesario intensificar las modificaciones en el estilo de vida y considerar la terapia farmacológica si aún no se ha indicado. Una vez que se inicia el tratamiento farmacológico, no está definido por cuánto tiempo debe continuarse y si está indicado suspenderse una vez que se normalizan los parámetros de control glucémico ( $\mathrm{HbA} 1 \mathrm{c}$, glucosa en ayuno), por lo que la decisión de si se debe continuar en estos casos debe individualizarse de acuerdo con el logro de las metas en otros componentes del tratamiento (plan de alimentación saludable, ejercicio y reducción de peso). Sin embargo, no debe abandonarse el seguimiento regular para reiniciar el tratamiento en caso de que se vuelva a presentar IG. EI objetivo de la prevención es retrasar la aparición de la diabetes y disminuir las complicaciones.

Es posible que los costos de la prevención estén en equilibrio con los costos ahorrados por el tratamiento de las complicaciones, ya que la conversión de la alteración de la glucosa alterada en ayuno a diabetes duplica el riesgo de mortalidad por enfermedad cardiovascular y la IG aumenta el riesgo de coronariopatía en un 50\%. El estudio DECODE (Diabetes Epidemiology Collaborative Analysis of Diagnostic Criteria in Europe ${ }^{22}$ demostró un riesgo mayor de cardiopatía isquémica en pacientes con hiperglucemia a las dos horas de la carga incluso con glucemia normal en ayunas. Adicionalmente se debe de realizar el tratamiento de otros factores de riesgo cardiovascular que frecuentemente coexisten en pacientes con prediabetes como control de presión arterial, colesterol de lipoproteínas de baja densidad y triglicéridos.

\section{RESULTADOS DEL TRATAMIENTO DE LA INTOLERANCIA A LA GLUCOSA EN PREVENCIÓN DE DIABETES Y REDUCCIÓN DEL RIESGO DE COMPLICACIONES MICROVASCULARES Y MACROVASCULARES}

En el estudio DPP se estableció que por cada kilo de peso perdido hubo una reducción del $16 \%$ en el riesgo de desarrollar DT2. En los pacientes que lograron la meta de ejercicio (150 minutos por semana) pero no la meta del peso, la reducción de desarrollar diabetes fue del $44 \%$ en comparación con el grupo control. En el estudio de seguimiento del DPP a 15 años $^{23}$, los pacientes que lograron normoglucemia en algún punto del seguimiento tuvieron menor riesgo de diabetes en comparación con los que no lograron.

Está claro que es posible prevenir la aparición de la diabetes en individuos con alto riesgo y cuantos más factores de riesgo estén presentes, más útil será la intervención oportuna. El mayor interés existe acerca de si el tratamiento de la prediabetes puede impactar favorablemente en la reducción de desenlaces, en especial infarto de miocardio, mortalidad cardiovascular y mortalidad de todas las causas. El estudio DPPOS (Diabetes Prevention Program Outcome Study) que es una extensión del DPP, evaluará tal efecto y se aguardan los resultados. Algo de la información que se desprende del análisis de este estudio se publicó en 2017 y se observó que la mayoría de los componentes y el riesgo cardiovascular estimado a 10 años se redujo significativamente entre los sujetos que tuvieron glucosa en ayuno y poscarga normales al menos una vez durante las revisiones 
anuales respecto a quienes se mantuvieron en el rango de prediabetes o progresaron a diabetes ${ }^{24}$.

En el estudio ACE (Acarbose Cardiovascular Evaluation) $)^{25}$, realizado en China, cerca de 6,500 pacientes con enfermedad coronaria e IG fueron asignados a recibir acarbosa $50 \mathrm{mg}$ tres veces al día o placebo, además de las medidas habituales de prevención secundaria. A los cinco años, el grupo asignado a acarbosa presentó menor incidencia de diabetes pero no tuvo ningún impacto en el MACE (major cardiovascular adverse event) de 5 puntos (muerte cardiovascular, infarto no fatal, enfermedad vascular cerebral no fatal, hospitalización por angina inestable y hospitalización por insuficiencia cardiaca). Se concluyó que en población china la acarbosa no reduce el riesgo de eventos cardiovasculares mayores.

\section{CONCLUSIÓN}

Se puede considerar a la prediabetes como un estado que puede incrementar el riesgo cardiovascular por su alta asociación con otros factores de riesgo. La búsqueda intencionada de prediabetes debiera ser una práctica habitual de los médicos y la aplicación de tratamiento, principalmente cambios en estilo de vida y metformina, debieran implementarse con mayor frecuencia. Existe evidencia suficiente acerca del impacto de diversas medidas sobre la reducción de incidencia de diabetes, así como de diversos factores de riesgo cardiovascular. La demostración del impacto del tratamiento de la prediabetes sobre variables de resultados duras (MACE 3, MACE 4, MACE 5) no ha sido aún confirmada, pero la evidencia indirecta sobre los factores subrogados pone sobre la mesa la conveniencia de su tratamiento temprano ${ }^{26}$.

\section{FINANCIAMIENTO}

La presente investigación no ha recibido ninguna beca específica de agencias de los sectores público, comercial, o sin ánimo de lucro.

\section{CONFLICTO DE INTERESES}

Los autores declaran no tener conflicto de intereses.

\section{RESPONSABILIDADES ÉTICAS}

Protección de personas y animales. Los autores declaran que para esta investigación no se han realizado experimentos en seres humanos ni en animales.

Confidencialidad de los datos. Los autores declaran que en este artículo no aparecen datos de pacientes.

Derecho a la privacidad y consentimiento informado. Los autores declaran que en este artículo no aparecen datos de pacientes.

\section{BIBLIOGRAFÍA}

1. American Diabetes Association. Classification and diagnosis of diabetes: Standards of medical care in diabetes 2019. Diabetes Care. 2019;42(Suppl. 1):S13-S28.

2. Robles-Osorio L, Aguilar-Salinas CA, Mehta R, Gómez-Pérez FJ, Rull JA. Analysis of fasting plasma glucose values for optimal detection of abnormal responses on the oral glucose tolerance test in at-risk study subjects. Endocr Pract. 2007;13:583-9.

3. McLaughlin T, Abbasi F, Cheal K, Chu J, Lamendola C, Reaven G. Use of metabolic markers to identify overweight individuals who are insulin resistant. Ann Intern Med. 2003;139(10):802-9.

4. Bartnik M, Rydén L, Malmberg K, Ohrvik J, Pyörälä K, Standl E, et al.; Euro Heart Survey Investigators. Oral glucose tolerance test is needed for appropriate classification of glucose regulation in patients with coronary artery disease: a report from the Euro Heart Survey on Diabetes and the Heart. Heart. 2007;93:72-7.

5. Huang Y, Cai X, Mai W, Li M, Hu Y. Association between prediabetes and risk of cardiovascular disease and all cause mortality: systematic review and meta-analysis. BMJ. 2016;355:i5953.

6. Vistisen D, Witte DR, Brunner EJ, Kivimäki M, Tabák A, Jørgensen ME, et al. Risk of cardiovascular disease and death in individuals with prediabetes defined by different criteria: The Whitehall II Study. Diabetes Care. 2018;41:899-906.

7. The Atherosclerosis Risk in Communities (ARIC) Study: design and objectives. The ARIC investigators. Am J Epidemiol. 1989;129:687-702.

8. The Diabetes Prevention Program. Design and methods for a clinical trial in the prevention of type 2 diabetes. Diabetes Care. 1999;22:623-34.

9. Tuomilehto J, Lindström J, Eriksson JG, Valle TT, Hämäläinen H, llanneParikka P, et al.; Finnish Diabetes Prevention Study Group. Prevention of type 2 diabetes mellitus by changes in lifestyle among subjects with impaired glucose tolerance. N Engl J Med. 2001;344:1343-50.

10. Knowler WC, Fowler SE, Hamman RF, Christophi CA, Hoffman HJ, Brenneman AT, et al.; Diabetes Prevention Program Research Group. 10-year follow-up of diabetes incidence and weight loss in the Diabetes Prevention Program Outcomes Study. Lancet. 2009;374:1677-86.

11. Haw JS, Galaviz KI, Straus AN, Kowalski AJ, Magee MJ, Weber MB, et al. Long-term sustainability of diabetes prevention approaches: A systema- 
tic review and meta-analysis of randomized clinical trials. JAMA Intern Med. 2017;177:1808-17

12. Wilmot EG, Edwardson CL, Achana FA, Davies MJ, Gorely T, Gray LJ, et al. Sedentary time in adults and the association with diabetes, cardiovascular disease and death: systematic review and meta-analysis. Diabetologia. 2012;55:2895-905.

13. American Diabetes Association. Prevention or delay of type 2 diabetes: Standards of medical care in diabetes-2018. Diabetes Care. 2018; 41(Suppl 1):S51-S54.

14. Estrategia mundial sobre régimen alimentario, actividad física y salud [Internet]. Organización Mundial de la Salud [fecha de consulta: 6 de enero de 2020]. Disponible en: https://www.who.int/dietphysicalactivity/physical_activity_intensity/es/

15. Orchard TJ, Temprosa M, Goldberg R, Haffner S, Ratner R, Marcovina S, et al.; Diabetes Prevention Program Research Group. The effect of metformin and intensive lifestyle intervention on the metabolic syndrome: the Diabetes Prevention Program randomized trial. Ann Intern Med. 2005;142:611-9.

16. Gillies CL, Abrams KR, Lambert PC, Cooper NJ, Sutton AJ, Hsu RT, et al. Pharmacological and lifestyle interventions to prevent or delay type 2 diabetes in people with impaired glucose tolerance: systematic review and meta-analysis. BMJ. 2007;334:299.

17. Torgerson JS, Hauptman J, Boldrin MN, Sjöström L. XENical in the prevention of diabetes in obese subjects (XENDOS) study: a randomized study of orlistat as an adjunct to lifestyle changes for the prevention of type 2 diabetes in obese patients. Diabetes Care. 2004:27(1):155-61.

18. A Randomized, double-blind, placebo-controlled, parallel-group study to investigate the safety and efficacy of the co-administration of canagliflozin $300 \mathrm{mg}$ and phentermine $15 \mathrm{mg}$ compared with placebo for the treatment of non-diabetic overweight and obese subjects. Study phase 2 [Internet]. Yale University: The YODA Project. Disponible en: https:// yoda.yale.edu/nct02243202-randomized-double-blind-placebo-controlled-parallel-group-study-investigate-safety-and
19. le Roux CW, Astrup A, Fujioka K, Greenway F, Lau DCW, van Gaal L, et al.; SCALE Obesity Prediabetes NN8022-1839 Study Group. 3 years of liraglutide versus placebo for type 2 diabetes risk reduction and weight management in individuals with prediabetes: a randomised, doubleblind trial. Lancet. 2017;389(10077):1399-409.

20. Chiasson JL, Josse RG, Gomis R, Hanefeld M, Karasik A, Laakso M; STOPNIDDM Trail Research Group. Acarbose for prevention of type 2 diabetes mellitus: the STOP-NIDDM randomised trial. Lancet. 2002;359(9323):2072-7.

21. DeFronzo RA, Tripathy D, Schwenke DC, Banerji M, Bray GA, Buchanan TA, et al.; ACT NOW Study. Pioglitazone for diabetes prevention in im paired glucose tolerance. N Engl J Med. 2011;364(12):1104-15.

22. Glucose tolerance and mortality: comparison of WHO and American Diabetes Association diagnostic criteria. The DECODE study group. European Diabetes Epidemiology Group. Diabetes epidemiology: Collaborative analysis of diagnostic criteria in Europe. Lancet. 1999;354:617-21.

23. Long-term effects of lifestyle intervention or metformin on diabetes development and microvascular complications over 15-year follow-up: the Diabetes Prevention Program Outcomes Study. Lancet Diabetes Endocrinol. 2015;3:866-75.

24. Perreault L, Pan Q, Aroda VR, Barrett-Connor E, Dabelea D, Dagogo-Jack $S$, et al.; Diabetes Prevention Program Research Group. Exploring residual risk for diabetes and microvascular disease in the Diabetes Prevention Program Outcomes Study (DPPOS). Diabet Med. 2017;34:1747-55.

25. Holman RR, Coleman RL, Chan JCN, Chiasson JL, Feng H, Ge J, et al.; ACE Study Group. Effects of acarbose on cardiovascular and diabetes outcomes in patients with coronary heart disease and impaired glucose tolerance (ACE): a randomised, double-blind, placebo-controlled trial. Lancet Diabetes Endocrinol. 2017:5:877-86.

26. Theodorakis MJ, Coleman RL, Feng H, Chan J, Chiasson JL, Ge J, et al.; ACE Study Group. Baseline characteristics and temporal differences in Acarbose Cardiovascular Evaluation (ACE) trial participants. Am Heart J. 2018;199:170-5. 